\title{
HOW THE INNOVATION DIFFUSION MODELS FROM THE PAST CAN HELP US TO EXPLAIN MARKETING IN THE NEW MEDIA ERA
}

\author{
Cinzia Colapinto, Ca' Foscari University of Venice, Italy. \\ Elena Sartori, Ca' Foscari University of Venice, Italy. \\ Marco Tolotti, Ca' Foscari University of Venice, Italy.
}

\begin{abstract}
Even if the rhetoric of the Internet and the new digital media seems to have radically changed our technological environment, historical recurrences are relevant tools in order to analyze the future marketing. We propose a new multi-stage model able to bridge two different approaches, namely the adoption models à la Bass and the recent line of research concerning agentbased innovation diffusion models. Our technology allows us to find a closed form equation for awareness and adoption, taking into account heterogeneous population.
\end{abstract}

Keywords: innovation diffusion, social media marketing, interacting agents, stochastic models.

\section{INTRODUCTION}

Historically companies (advertisers) have thought of themselves as top-down communicators, in control of what information is released, to whom, how and when (Varey 2002). Thanks to new media and Internet, advertising has become more about connections and share ideas. The speed and ease of communicating via the Internet have, indeed, created a new and complex scenario: websites use technical translation strategies, blogs are associated with emotional support functions, and social media establish dialogues with stakeholders (Stephens and Malone 2010). In particular, new and social media are fastmoving phenomena, powerful new communication platforms capable of underpinning marketing and communication strategies. In the last decade, online communications have influenced how organizations share messages with the media and the public, as it is imperative to conduct timely, accurate and effective communication exchanges in the information society (Taylor and Perry 2005). The new scenario has, also, affected the personal communication among peers, thus diffusion is becoming more and more a social process, involving interpersonal communication relationships. In other words, in the socalled knowledge society information and innovation are crucial. In particular, it is relevant the diffusion of innovations, which is a process related to the spread of a new idea, product or technology through the members of a social system. Rogers (1962) defines diffusion as "the process by which an innovation is communicated through certain channels over time among the members of a social system". Moreover, we agree with the new definition of the creative industries in terms of social network markets, where both demand and supply operate in complex social networks (Potts et al. 2008).

If we take stock of the last decades and think about the next decade and beyond, we can learn from the past to better navigate the marketing future. The aim of this paper is to review and extend our knowledge base concerning innovation diffusion. Indeed, from a marketing perspective it is relevant to understand how the information flow, through mass media and/or among peers, affects the adoption decisions of consumers and the diffusion of a new product or service. Many researchers have studied how ideas propagate through a society (Rogers 1962) or through a well-defined network structure (see the spatial model by Goldenberg et al. 2009), or how marketing mix strategies affect new product diffusions (see, e.g., Bass et al. 1994). We would like to provide a new multi-stage model able to merge different approaches and to give a formal modelization to previous empirical results.

The structure of the paper is as follows. Section 2 presents the rationale behind our model: through a literature review; we identify and illustrate the two main literature streams on which we base our model. In Section 3 we provide our multi-stage model. Section 4 concludes.

\section{DRAWING FROM THE PAST}

In this section we point out the roots of our model; our aim is to build a more comprehensive framework able to capture some of the typical features of the new diffusion channels; we develop a multistage-stage diffusion process and introduce suitable connectivity levels (network structure). Moreover, we take into account emotion driven motives to the adoption and allow for heterogeneity in the attitudes of the actors.

We identify two main lines of research dealing with innovation diffusion. The first refers to the famous Bass (1969) model and the second, and more recent stream, to agent-based models (ABM). If Bass (1969) represents a crucial contribution to the 
field of innovation diffusion at the aggregate level, agent-based models look at micro-level variables affecting the innovation diffusion process. Obviously, these two mentioned streams are not in opposition. The micro-formalization of how a consumer decides and behaves is the foundation to understand market penetration at the aggregate level: think at Micromotives and Microbehavior à la Schelling (1978).

In our model we, also, recall the adoption process, the classical normal distribution model, introduced by Rogers (1962). Starting from several empirical studies, Rogers detects features and mechanisms typical of the diffusion process and points out different steps through which the innovation decision process develops: knowledge, persuasion, decision, implementation and confirmation. The gap between awareness and adoption persists in recent papers, such as in Fanelli and Maddalena (2012), who use a delayed equation to analyze at the aggregate level, without addressing the decision rationale behind it.

In this process the features of the decision maker (socio-economic characteristics, personality variables and communication behavior) are important and affect the choice between adoption or rejection. Rogers, and previously Ryan and Gross (1943), also identifies five different adopters categories as ideal types, i.e., innovators (venturesome), early adopters (respectable), early majority (deliberate), late majority (skeptical) and laggards (traditional). We believe it is important to consider consumers as independent entities with heterogeneous preferences and behaviors capable of changes. The innovativeness defined by Rogers has also been modified and extended by Hirschman (1980), who talks about adoptive and vicarious innovativeness and creative consumers.

\section{Aggregate Level Models: The Bass Framework}

Bass proposes an analytical model for the timing of initial purchase of new products, paying particular attention to the behavioral rationale of consumers behind the adoption decision. In a fully connected and homogenous population, he distinguishes two main sub-groups: 'innovators', which adopt early the new product independently of the others, and 'imitators' (all which in the classification of Rogers are not innovators as shown by Mahajan et al. 1990). A consumer adopts/buys the new product because of two different influences: external influences ( $p$, the coefficient of innovation), such as advertising and other communications (mass-media communication) initiated by the firm (subsuming the model introduced by Fourt and Woodlock 1960), and internal market influences ( $q$, the coefficient of imitation) that result from interactions among adopters and potential ones in the population (word of mouth as in Mansfield 1961). The model shows the wellknown S-shaped cumulative adoption curve.

A limit of the Bass model is that, in order to maintain tractability, it shrinks the whole (complex) diffusion process into a single adoption curve. Despite its simplicity (or maybe thanks to it), the Bass model is still the reference for the analysis of the diffusion of new technologies even in the literature concerning the marketing applied to new media. To better capture some important issues related to the diffusion of innovations, many authors have developed the Bass framework in several directions. Among them we can mention: Mahajan and Peterson (1985), who extend and simplify the Bass model, and Norton and Bass (1987), who consider subsequent generations of innovation in order to make the adoption curve more persistent.

Traditional aggregate models are not behaviorally based, thus they do not reproduce the complexity of real-world diffusion patterns. Additionally, the mathematical form of the Bass model requires the assumption that the potential adopter population is homogeneous; on the contrary, the heterogeneous population argument was already used by Rogers. These limits explain why we prefer to look also at agent-based models.

A recent work dealing with social contagion is the one by Van den Bulte and Joshi (2007). The authors point out that marketing diffusion models need to assume heterogeneity to study the viral emerging practice. They model the aggregatelevel diffusion path focusing their attention at the networks connecting consumers: they allow for heterogeneity in the population's characteristics, using two adopter segments (influentials and non influentials agents).

\section{The Micro-Foundation: Agent-Based Models}

This approach is based on the idea that phenomena at the aggregate level can be understood as emerging from interactions between individuals at the micro level. Indeed, agent-based models focus on an individual customer, who maximizes some objective function (utility or benefit from the product) according to his/her perceptions of the innovation's performance, value or benefit. These models can capture micro-level individuals' underlying decision processes and mimic dynamic social effects observed at the macro level in the marketplace. However, researchers provide interesting simulations losing the capability of abstraction of an aggregate model.

As shown by Kiesling et al. (2011), most of researchers model the micro-motives behind the decision process, introducing specific characteristics of single actors that are difficult to be captured by means of a macro/aggregate equation. The price they pay is the loose of tractability and the need of numerical methods, in order to provide intuition about dynamics and equilibria. In ABM, such as Delre et al. (2010), the decision-making process is guided by a weighted utility of individual preference and social influence. It means that the total utility of consuming a new product is composed of a social utility part 
and an individual one. In this way the weights in the model can describe both individualistic agents and socially susceptible ones, meaning that agents' utility depends on what the neighbors do.

The most famous example of social agent-based simulation has been developed by Schelling (1978), who demonstrates that racial segregation can emerge in communities from the behaviors of autonomous agents, each individually exhibiting very small racial prejudices. Schelling (1978:13) explores the relation between the behavioral characteristics of the individuals, who comprise some social aggregate and the characteristics of the aggregate.

Recently, Goldenberg et al. (2009) examine both the aggregate growth process and what happens at the individual level in a network. They use an ABM to study dynamics in a well-documented social network. In particular, they concentrate on the role of hubs, i.e., highly interconnected individuals in a social network, in the adoption process, and statistically test different hypotheses concerning it. They point out that a hub's customer value has to include the effect on others.

Focusing on individual-level psychological processes, Berger and Milkman (2012) show that emotions whose content evokes in individuals, help determine which cultural items succeed in the marketplace of ideas, that is at the macro-level. The relation between emotion and diffusion or virality is very complex and relevant in the marketing perspective.

\section{THE MODEL}

We consider a population of $N$ possible adopters, linked with each other by a social network. We monitor the diffusion process of an innovation (product, service, idea or content) launched through the network. We identify two crucial steps: (i) an actor has to become aware of the existence of the innovation (awareness); (ii) he/she engages a personal decision process, eventually leading to definitive adoption (adoption). Under this modeling idea we are referring to the seminal Rogers' diffusion process (see Rogers 1962). We see that, in doing this, the formal model we are proposing, allows to take into account features that are specific of the new communication channels. Henceforth, our aim is to reinterpret Rogers in view of the digital era.

More into details, to model the awareness dynamics we take into account a proxy of the connectivity degree of each agent (at the micro level) and an aggregate statistics of the spread of the information on the network (at the macro level), corresponding to the proportion of agents already informed. To formalize it, let, first, define the awareness process $\boldsymbol{x}(t)=$ $\left\{\left(x_{1}(t), \ldots, x_{N}(t)\right), t \geq 0\right\}$, where $\forall i, x_{i}(t) \in\{0,1\} . x_{i}(t)=0$ means that, up to time $t$, actor $i$ has not been reached by the information; if reached, $x_{i}(t)=1$.

Dealing with diffusion through a social network, we assume that the knowledge spreads by (informational) contagion. This viral attitude is testified in many empirical works (Berger and Milkman 2012, Phelps et al. 2004). From a modeling viewpoint, following recent literature on contagion applied to a social environment (see, for instance, Dai Pra and Tolotti 2009 and Barucci and Tolotti 2012), we assume that agent $i$ becomes aware at a random time $\tau_{i}$. We suppose that $\tau_{i}$ is exponentially distributed with parameter $\lambda_{i}^{x}=\exp \left(\beta_{i} m_{N}^{x}\right)$, where $\beta_{i} \geq 0$ measures the personal connection degree (connectivity), and $m_{N}^{x}(t)=\frac{1}{N} \sum_{i=1}^{N} x_{i}(t)$ is the aggregate statistics we consider to represent the proportion of agents already aware. Notice that, the more an agent is interconnected, the higher is the probability of becoming aware.

To model the adoption-dynamics we consider, in particular, the propensity to adopt of each agent, his/her inclination to conform and the actual level of adoptions, i.e., the number of adoptions already occurred. Once reached by the information, agent $i$ can decide whether to adopt or not.

So, we define the adoption process $\boldsymbol{y}(t)=\left\{\left(y_{1}(t), \ldots, y_{N}(t)\right), t \geq 0\right\}$, where $\forall i, y_{i}(t) \in\{0,1\} . y_{i}(t)=0$ means that, up to time $t$, actor $i$ has not adopted the new product, otherwise $y_{i}(t)=1$.

Let denote, moreover, by $p_{i} \geq 0$ and $q_{i} \geq 0$ the parameters describing, respectively, the propensity to adopt of agent $i$ and his/her attitude to conform, and by $m_{N}^{y}(t)=\frac{1}{N} \sum_{i=1}^{N} y_{i}(t)$ the actual proportion of adopters, hence, the aggregate statistics characterizing adoption. We have introduced all the relevant parameters and variables of our model. To summarize, we associate to each agent an identity vector, which comprises the personal characteristics that can influence him/her in the whole diffusion process, defined as follows.

Definition. For $i=1, \ldots, N$, let $\theta_{i}=\left(\beta_{i}, p_{i}, q_{i}\right)$ be the identity vector of actor $i$, where $\beta_{i}$ is the connectivity, $p_{i}$ the innovation coefficient and $q_{i}$ the imitation coefficient. $\theta_{i}, i=1, \ldots, N$, are independent copies of a random vector $\theta=$ $(\beta, p, q)$ with distribution $\eta_{\theta}$.

As already said, once aware, an actor decides whether to adopt according to some utility. To maintain tractability and to mimic the adoption rationale behind the Bass (1969) model and subsequent literature, we assume that at time $t \geq \tau_{i}$ agent $i$ decides to adopt when

$p_{i}+q_{i} m_{N}^{y}(t) \geq c_{i}$ 
where $c_{i} \geq 0$ is a random threshold. We assume that $\forall i, c_{i}$ are i.i.d. random variables uniformly distributed on the interval $[0,1] . c_{i}$ is intended to summarize all those aspects that are not related to the identity of the agent, but that are functional to the adoption itself (prices, external factors, personal environment). For more details and for a more general presentation we refer the reader to Colapinto et al. (2012).

To ease the intuition, we represent the rationale behind the diffusion process described above by means of the diagram reported in Figure 1. Time $\tau_{i}$ represents the (random) time at which actor $i$ is reached by the information about the innovation. Note that, before $\tau_{i}$, he/she cannot even take into consideration the possibility of adopting. At subsequent exponentially distributed random times $T_{i}^{1}, \ldots, T_{i}^{K}$, agent $i$ will be asked whether he/she wants to adopt. Facing a binary decision problem, the answer can be YES or NO. Depending on this result, the variable $y_{i}$ remains 0 or is turned into 1 . Once adopted, the agent cannot change his/her mind. We stress the fact that, according to our scheme, we are able to reproduce a complex decision process that encompasses many of the Rogers' phases (see Rogers (1962)). In particular, we recognize persuasion (the actor feels peer pressure via the social component of his/her utility), decision (at times $T_{i}^{K}$ ) and confirmation (the first time in which he/she decide to confirm his/her choice).

We are, now, ready to state the theorem which explicitly shows the equations describing the awareness and the adoption processes. To this aim, we need to introduce still a couple of technical objects. We note, in particular, that, from a technical viewpoint, the process we have describe is a Markov process taking values on the space $\{0,1\}^{2 N}$, uniquely defined by means of two families of transition rates $\left(\lambda_{i}^{x}, \lambda_{i}^{y}\right)$, where

$\lambda_{i}^{x}=\exp \left[\beta_{i} m_{N}^{x}\right]$,

$\lambda_{i}^{y}=x_{i}\left[p_{i}+q_{i} m_{N}^{y}\right]$.

More details can be found in Colapinto et al. (2012).

Theorem. Consider the Markov process $(\boldsymbol{x}(t), \boldsymbol{y}(t))$ with transition rates $\left(\lambda_{i}^{x}, \lambda_{i}^{y}\right)$ and assume, at time $t=0$, $x_{i}(0)=$ $y_{i}(0)=0$, for $i=1, \ldots, N$.

Then, for $N \rightarrow \infty,\left(m_{N}^{x}(t), m_{N}^{y}(t)\right)$ weakly converge to $\left(m^{x}(t), m^{y}(t)\right)$, where

$m^{x}(t)=E^{\theta}\left[m^{x}(t ; \theta)\right]$

$m^{y}(t)=E^{\theta}\left[m^{y}(t ; \theta)\right]$

and

$\dot{m}^{x}(t ; \theta)=\left(1-m^{x}(t ; \theta)\right) \exp \left[\beta m^{x}(t)\right]$

$\dot{m}^{y}(t ; \theta)=\left(m^{x}(t ; \theta)-m^{y}(t ; \theta)\right)\left(p+q m^{y}(t)\right)$.

Here, $E^{\theta}$ denotes the average taken with respect to the distribution $\eta_{\theta}$ of $\theta=(\beta, p, q)$.

The result is very technical and difficult to interpret at a first glance. However, notice that, putting $x_{i} \equiv 1 \forall i$ (i.e., looking only at adoption without network effects) and considering $p_{i} \equiv p$ and $q_{i} \equiv q \forall i$ (i.e., considering a homogeneous population), we recover exactly the original Bass (1969) model. Indeed, under these assumptions, Equations (3) reduce simply to

$\dot{m}^{y}(t)=\left(1-m^{y}(t)\right)\left(p+q m^{y}(t)\right)$.

Note, in fact, that the first equation in (3) is simply $\dot{m}^{x}(t ; \theta)=0$, since $x_{i} \equiv 1, \forall i$. Equation (5) gives rise exactly to the Bass adoption curve.

A better understating of the result can be given by a representative application. In the following section, we see how in a very specific example, it is possible to recover important features concerning the multi-stage diffusion process empirically found already in Rogers (1962), but reinterpreted in the context of new communication channels.

\section{Innovators and Hubs}

...there is no evidence that personal innovativeness is correlated with social connectivity. Goldenberg et al.(2009).

One significant application of our technology can be used to give a modeling support to the intuition reported in the above quotation taken by Goldenberg et al. (2009). As already discussed in Section 2, in that paper, the authors empirically analyze the effects of two very important classes of actors: hubs and innovators. One of their main results is to empirically show that the role of these two typologies of agents have to be disentangled (as opposed to many recent papers in the field, where the concept of hub and innovative people is often merged in the concept of opinion leader). Arguing on this, we apply our model to a population, where actors are divided into four sub-populations of homogeneous agents with different proportions. More specifically, we consider innovator/hub, innovator/non-hub, follower/hub and follower/non-hub, as shown in Figure 2. Since our actors are characterized by means of an identity vector $\theta=(\beta, p, q)$, in this example we have to specify four different 
identity vectors (one for each sub-population). Note that we have three important dimensions (connectivity, innovativeness and imitative behavior). Concerning connectivity, it is related to awareness and is represented by the parameter $\beta$. Innovativeness and imitative behavior are represented by $p$ and $q$ (as in Bass 1969) and are assumed to play "opposite" roles: if $p$ is high, then $q$ is low and vice versa.

The advantage of a simplified world with four sub-groups makes Equations (3)-(4) more tractable. In particular, the awareness and adoption curves can be precisely drawn. In Figure 3 (left panel) we plot the two curves (awareness and adoption) under specifications as in Figure 2. As a matter of fact, we can see that many agents, although made aware of the innovation, may need some time to be convinced about the adoption. These results in a time delay of the adoption curve as already shown by empirical evidence in Rogers (1962) (see left panel of Figure 2 taken by Rogers 1962). What is interesting to notice, is the fact that, according to our simulation, the awareness curve (blue dotted line in the left panel) and the adoption curve (black continuous line), can exhibit very different shapes: an exponential growth the former, a more S-shaped character the latter. Note that the spread of knowledge through new communication channels is often of an exponential type (see Pousttchi and Wiedemann 2007, Kaplan and Haenlein, 2011). This fact reinforces our intuition that, although very rapid in diffusing awareness, the new media may still suffer of a slower adoption curve.

A preliminary comparative statics analysis on the parameters of the model shows that: (i) increasing $q$ makes the adoption curve more close to the awareness one; (ii) increasing $q$ for both innovators and followers has an higher effect compared to increasing $q$ for just innovators or followers; (iii) the impact of $p$ is less pronounced than the effect of $q$ on the adoption curve. From a managerial point of view, this implies that, in order to foster the adoption process, decision makers have better to stimulate the imitation process, as the increase in $q$ can be driven by an effort in pushing up the viral effect of the campaign itself. Points (i) and (iii), thus, suggest that in the context of new media, it is more rewarded to invest on a social message compared to a classical (personal) advertisement. Instead, (ii) points out that once a critical mass of innovators (and possibly of "hub-type" ones) has been convinced, it is more efficient to invest equally on the remaining innovators and on the bigger population of late-adopters.

\section{CONCLUSIONS}

The multi-stage model we present acts as a bridge between two main lines of research in innovation diffusion. It recalls the Bass-style model, but it also includes the complexity introduced by Rogers: we maintain tractability introducing a closed form equation able to explain the process as well. Moreover, the technology allows us to characterize the agents (preferences, attitudes, social capital, private and social utility). Drawing from the past, we present a model able to describe previous empirical results in the new media environment. A hot issue is the real value of social and new media in sustaining the launch of a new product. Our model confirms the hypothesis that new media can increase awareness more than adoption of a new product (see Harris and Rae 2009). Some managerial implications are drawn from this empirical evidence.

Future directions of research concern the analysis of empirical data, useful to fit the parameters of the model and infer good practices in advertising innovation through the new communication channels. A limit of our model is that we do not formalize a specific network structure that represents different market characteristics; obviously, a structure may affect the final success of a new product that enters the market. However, we introduce the degree of connectivity as a good proxy in a social media context.

Figure 1: The diffusion process for actor " $i$ " as described by our model

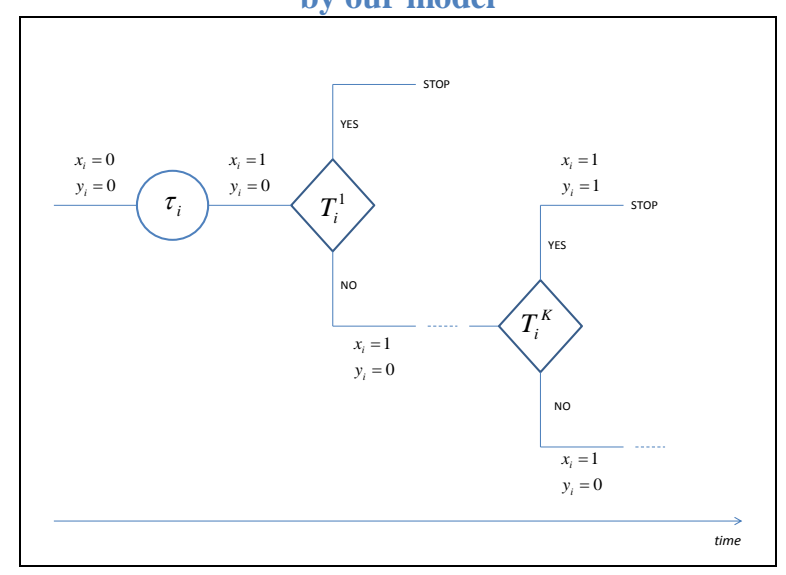

Figure 2: Identity vectors of the four sub-populations

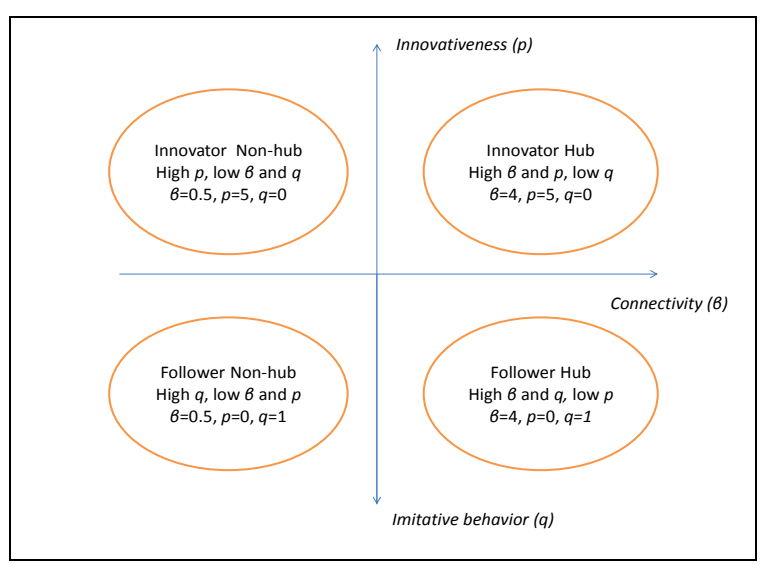


Figure 3: Awareness versus Diffusion. Our model (left) and Rogers' evidence from data (right)
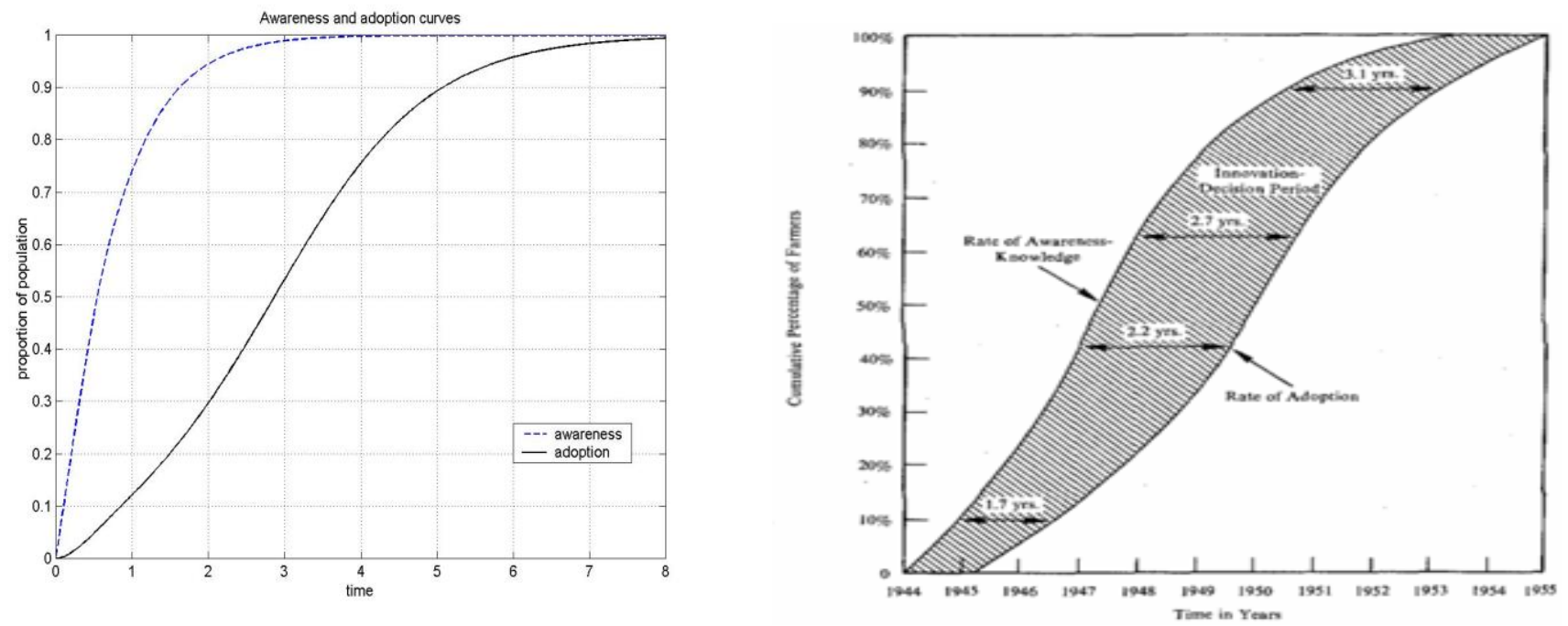

\section{REFERENCES}

Barucci, E. and Tolotti, M. (2012). Social interaction and conformism in a random utility model. Journal of Economic Dynamics \& Control 36 (12): 1855-1866.

Bass, F. M. 1969. A new product growth for model consumer durables. Management Science 15 (5): 215-227.

Bass, F. M., Krishnan, T. V. and Jain, D. C. (1994). Why the Bass model fits without decision variables. Marketing Science, 13(3), 204-223.

Berger, J. and Milkman, K. L. (2012). What Makes Online Content Viral?. Journal of Marketing Research, 49(2), 192-205.

Blume, L. and Durlauf, S. (2003). Equilibrium concepts for social interaction models. International Game Theory Review, 5(3), 193-209.

Brock, W. and Durlauf, S. (2001). Discrete choice with social interactions. Review of Economic Studies, 68(2), 235-260.

Colapinto, C., Sartori, E. and Tolotti, M. (2012). A two-stage model for diffusion of innovations. Mimeo.

Dai Pra, P. and Tolotti, M. (2009). Heterogeneous credit portfolios and the dynamics of the aggregate losses. Stochastic Processes and their Applications, 119(9), 2913-2944.

Delre, S. A., Jager, W., Bijmolt, T. H. A. and Janssen, M. A. (2010). Will it Spread or Not? The Effects of Social Influences and Network Topology on Innovation Diffusion. Journal of Product innovation management, 27, 267-282.

Fanelli, V. and Maddalena, L. (2012). A time delay model for the diffusion of a new technology. Nonlinear Analysis: Real World Applications, 13, 643-649.

Fourt, L.A. and Woodlock, J. W. (1960). Early Prediction of Market Success of Grocery Products. Journal of Marketing, 25, 31-38.

Goldenberg, J., Han, S., Lehmann, D. R. and Hong, J. W. (2009). The role of hubs in the adoption process. Journal of Marketing, 73(2), 1-13.

Harris, L. and Rae, A. (2009). Social networks: the future of marketing for small business. Journal of Business Strategy, 30(5), 24-31.

Hirschman, E.C. (1980). Innovativeness, Novelty Seeking and Consumer Creativity. Journal of Consumer Research, December, 283-295.

Kaplan, A.M. and Haenlein, M. (2011). Two hearts in three-quarter time: How to waltz the social media/viral marketing dance. Business Horizons, 54(3), 253-263.

Katz, E. and Lazarsfeld, P. F. (1955). Personal Influence. New York: The Free Press.

Kiesling, E., Güunther, M., Stummer, C. and Wakolbinger, L. M. (2011). Agent-based simulation of innovation diffusion: a review. Central European Journal of Operations Research, Physica Verlag, 1-48.

Mahajan, V., Muller, E. and Srivastava R. K. (1990). Determination of Adopter Categories by Using Innovation Diffusion Models. Journal of Marketing Research, XXVII(February), 37-50. 
Mahajan, V. and Peterson, R. A. (1985). Models for Innovation Diffusion. Sage University Paper Series on Quantitative Applications in the Social Sciences, No. 07-048. Sage Publications.

Mansfield, E. (1961). Technical Change and the Rate of Imitation, Econometrica, 29, 741-766.

Norton, J. A. and Bass, F. M. (1987). A diffusion theory model of adoption and substitution for successive generations of high-technology products. Management Science, 33(9), 1069-1086.

Parker, P. and Gatignon, H. (1994). Specifying competitive effects in diffusion models: An empirical analysis. International Journal of Research in Marketing, 11(1), 17-39.

Peres R., Muller, E. and Mahajan V. (2010). Innovation diffusion and new product growth models: A critical review and research directions. International Journal of Research in Marketing, 27, 91-106.

Phelps, J. E., Lewis, R., Mobilio, L., Perry, D. and Raman, N. (2004). Viral Marketing or Electronic Word-of-Mouth Advertising: Examining Consumer Responses and Motivations to Pass Along Email. Journal of Advertising Research, 44(4), 333-348.

Potts J., Cunningham, S., Hartley J. and Ormerod, P. (2008). Social network markets: a new definition of the creative industries. Journal of Cultural Economics, 32, 167-185.

Pousttchi, K. and Wiedemann, D. G. (2007). Success Factors in Mobile Viral Marketing: A Multi-Case Study Approach. Proceedings of the 6th International Conference on Mobile Business (ICMB 2007). IEEE Computer Society Press. Toronto, Ontario, Canada.

Rogers, E. M. (1962). Diffusion of Innovations. New York: The Free Press.

Ryan, B. and Gross, N.C. (1943). The Diffusion of Hybrid Seed Corn in Two Iowa Communities. Rural Sociology, 8, $15-24$. Schelling, T. C. (1978). Micromotives and Macrobehavior. W.W. Norton \& C.

Stephens, K. K. and Malone, P. (2010). New Media for Crisis Communication: Opportunities for Technical Translation, Dialogue, and Stakeholder Responses. In: Coombs, W.T. and Holladay, S.J. (eds), The Handbook of Crisis Communication. Chichester: Wiley-Blackwell, 381-395.

Taylor, M. and Perry, D. C. (2005). Diffusion of traditional and new media tactics in crisis communication. Public Relations Review, 31, 209-217.

Van den Bulte, C. and Joshi, Y. V. (2007). New product diffusion with influentials and imitators. Marketing Science, 26(3), 400-421.

Varey, A. (2002). Marketing Communication. Principles and practice. London, New York: Routledge. 